\title{
Behavior of HepG2 liver cancer cells using microfluidic-microscopy: A preliminary study
}

\author{
Hande Karamahmutoglu*a ${ }^{*}$, Metin Cetin ${ }^{\mathrm{b}}$, Tamer Yagci ${ }^{\mathrm{b}}$, Meltem Elitas ${ }^{\mathrm{a}}$ \\ ${ }^{\mathrm{a}}$ Biomechatronics Laboratory, Sabanci University, 34956, Istanbul, Turkey; ${ }^{\mathrm{b}}$ Dept. of Molecular \\ Biology and Genetics, Gebze Technical University, 41400, Kocaeli, Turkey
}

\begin{abstract}
Hepatocellular carcinoma is one of the most common types of liver cancer causing death all over the world. Although early-stage liver cancer can sometimes be treated with partial hepatectomy, liver transplantation, ablation, and embolization, sorafenib treatment is the only approved systemic therapy for advanced HCC. The aim of this research is to develop tools and methods to understand the individuality of hepatocellular carcinoma. Microfluidic cell-culture platform has been developed to observe behavior of single-cells; fluorescence microscopy has been implemented to investigate phenotypic changes of cells. Our preliminary data proved high-level heterogeneity of hepatocellular carcinoma while verifying limited growth of liver cancer cell lines on the silicon wafer.
\end{abstract}

Keywords: Microfluidics, HepG2, heterogeneity, microscopy, single-cell analysis, silicon wafer.

\section{INTRODUCTION}

Primary liver cancer is one of the most lethal tumor types in the world with approximately one million cases per year [1]. Hepatocellular carcinoma (HCC), as a histological type of primary liver cancer, has poor systemic molecular targeted therapies, which has been admitted widely due to enormous intertumor and intratumor heterogeneity in HCC [1,3]. Although, partial hepatectomy, liver transplantation, ablation and embolization, sorafenib treatment (Nexavar, Bayer HealthCare Pharmaceuticals) can sometimes cure early-stage liver cancer [4,5], still our understanding of its heterogeneity and treatment of liver cancer is at its crawling stage. There are several mechanisms that drive tumor heterogeneity such as etiological and environmental factors, patient ethnicity, cancer stem cells, and diversity in genetics and epigenetics [6].

Various methods ranging from genomic profiling to functional analyses have been implemented to understand the heterogeneity of liver cancer [1]. However, development of novel tools and methods to better understand cellular individuality and quantitatively characterize heterogeneity of HCC is still crucial. Hence, single-cell assays might compensate the limitations of bulk assays such as averaging the entire population and masking rare phenotypes [7]. Moreover, these assays might provide direct and quantitative readouts instead of averaging measurements. Microfluidic technologies in combination with advanced imaging systems present promising tools in this area. These tools are eligible to culture cells using well-controlled growth conditions and obtaining real-time measurements [8-9]. Besides, these modern methods reduce the assay time, sample consumption and waste production, while improving the throughput and precision.

In this study, we developed a microfluidic platform, which allows growth and real-time observation of HepG2 liver cancer cells using inverted fluorescent microscopy. Our preliminary results were consistent with [10], liver cancer cells exhibited limited growth on silicon wafer.

\section{METARIALS AND METHODS}

\subsection{Reagents and cell culture in batch}

HepG2 liver cancer cells, provided by Gebze Technical University Molecular Biology and Genetics department, were used to demonstrate the utility of the microfluidic cell culture platform. These cells were cultured in DMEM supplemented (PAN-Biotech) with 10\% FBS (Sigma-Aldrich) both in 75cm2-flasks (Corning ${ }^{\circledR}$ T-75 flasks) and in the 
microfluidic chips inside the incubator (NUVE EC160) where $37{ }^{\circ} \mathrm{C}, 5 \% \mathrm{CO} 2$ and humidity were provided. TrypsinEDTA solution (Sigma-Aldrich) was used to detach the cells from the flask and to load those cells into the microfluidic chips.

\subsection{Microfluidic device fabrication}

The designs were patterned on a thin film chromium deposited-photo mask (Cr-blank) using Vistec/EBPG5000plusES Electron Beam Lithography system. SU-8 3050 (SU-8® 3050, MicroChem) was spin coated on a 4-inches silicon wafer to obtain the structures with 50 - $\mu \mathrm{m}$ heights. Next, the photoresist-coated wafers were soft baked at $65{ }^{\circ} \mathrm{C}$ for 1 minute and at $95{ }^{\circ} \mathrm{C}$ for 5 minutes. Afterwards, they were exposed to UV light $(160 \mathrm{~mJ} / \mathrm{cm} 2)$ using the e-beam written masks by Midas/MDA-60MS mask aligner. Upon two consecutive post baking process at $65{ }^{\circ} \mathrm{C}$ for 1 minute and at $95{ }^{\circ} \mathrm{C}$ for 5 minutes, they were developed for 5 minutes using MicroChem's SU-8 developer. Later, they were rinsed with isopropanol.

The wafers were used as molds for the elastomeric polymer, polydimethysiloxane (PDMS, Sylgard® 184, Dow Corning, Midland, MI). PDMS base and curing reagents were mixed at 10:1 ratio. Upon bubble removal through vacuuming for 30 minutes, the mixture was baked at $75{ }^{\circ} \mathrm{C}$ for 60 minutes. Then, the PDMS devices were cut and the inlet and outlet ports were cut. Finally, the PDMS chips were bonded to a glass slide using Corona system (BD20-AC, Electro-Technic Products Inc.).

\subsection{Cell culture in microfluidic device}

Before cell loading into the microfluidic devices cell culture platform, metal couplers and tips couplers were autoclaved (Hirmaya (HMC) HV-85L) and tips were attached to medium inlet and outlet ports. Prior to usage, the medium was warmed at $37-^{\circ} \mathrm{C}$ water bath. HepG2 cells were centrifuged at $1800 \mathrm{rpm}$ for 10 minutes in $15 \mathrm{ml}$ falcons. Next, the supernatant was removed, and the pellet was completed to $1 \mathrm{ml}$ with fresh DMEM with \%10 FBS. Then, the cells were counted with hemocytometer and loaded into the device by a pipetman, connected to a blunt needle with a modified tip, with approximately 100000 cells $/ \mathrm{ml}$. The chambers were filled with cells through the cell trapping mechanism in single cell resolution. The microfluidic platform was placed into an autoclaved glass beaker with tissues wetted with phosphate buffered saline (PBS, Sigma-Aldrich) to prevent medium evaporation from the PDMS device. After microscopy inspection (Carl Zeiss, Primovert Model Trinocular Inverted Microscope) of the cells in the microfluidic cell culture platform, the device was transferred into the incubator overnight.

\subsection{Microscopy and image analysis}

The images of the individual chambers within the devices were captured using 10x objective using a Carl Zeiss, Axio Observer Z1 motorized stage equipped with the AxioCam Mrc5 camera. The cell images were obtained using $12.5 \mathrm{~ms}$ exposure under transparent light. Upon imaging, we manually counted the number of cells with and without pseupodium.

\section{RESULTS}

We developed our PDMS-glass device using soft lithography [11]. Figure 1 shows the design and dimensions of the device.

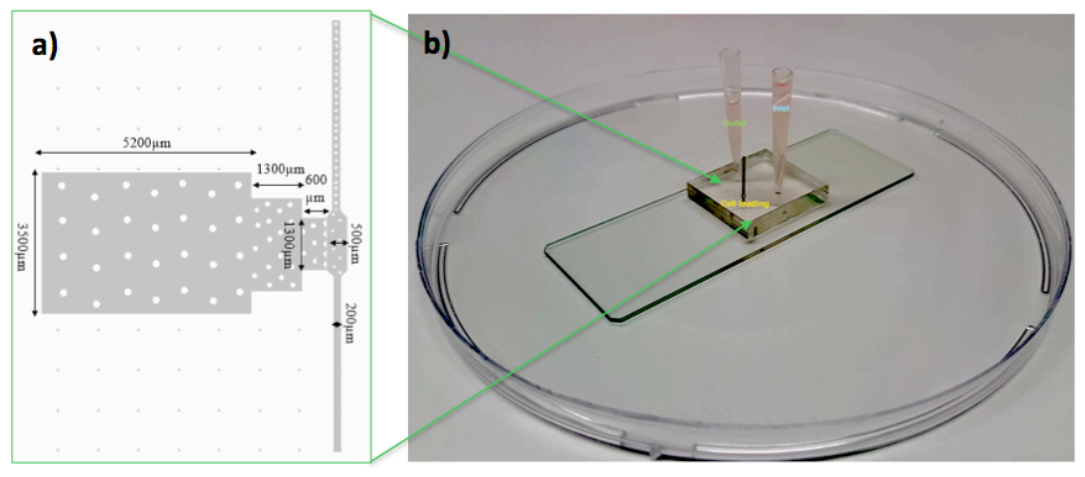

Figure 1. Design of the microfluidic chip with dimensions (a) and an image of the operating device (b). 
Growth of the hepatocellular carcinoma cell line is observed both at population-level using batch culture and at singlecell level using microfluidic-microscopy system above. Figure 2 shows growth of the hepatocellular cells over night at batch culture and microfluidic device.
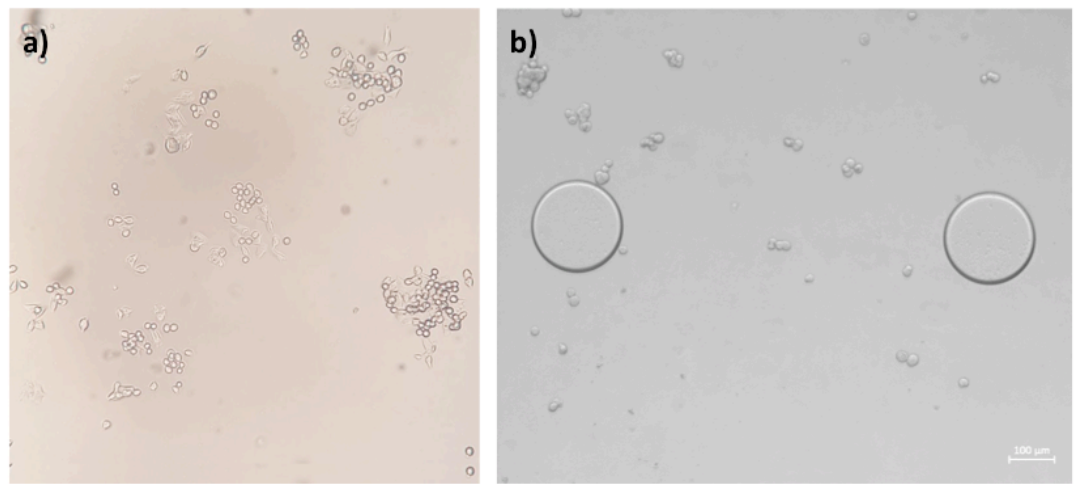

Figure 2. Morphology of HepG2 cells in tissue culture petri dish (a) and PDMS-Silicon microfluidic device (b) overnight at $37^{\circ} \mathrm{C}$.

Both morphology images show heterogeneity of the cells; some cells have developed cellular pseupodium while some of them have stayed circular, and these cells either developed some pseupodium in later stage of their inoculation or they have never generated.

Figure 3 shows the number of single-cells those developed pseupodium after overnight growth both in batch culture and microfluidic device.
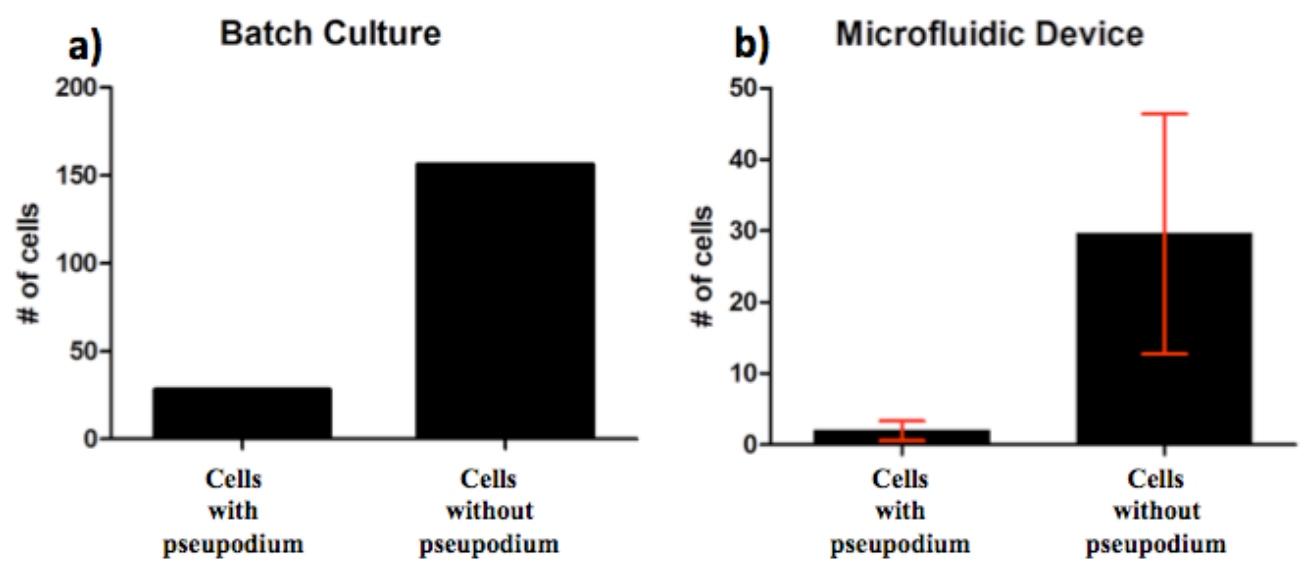

Figure 3. Single-cell analysis of liver cancer cells with and without pseupodium.

\section{DISCUSSION AND CONCLUSION}

Our preliminary result shows the morphological difference of overnight grown liver cancer cell lines in the tissue-culture petri dish and microfluidic device. The HepG2 cell line did not develop pseupodium when they were cultured in the PDMS-glass microfluidic device. Our preliminary results support the Xiaohui et al. [1]. When the cells did not grow pseupodium, they could not attach the surface of the glass and grow. They survived only a few days with the spherical morphology and they created cellular clusters. When we stained these clusters with DAPI and propodium iodide, we observed that most of the cells were dead. Therefore, when microfluidic technologies are developed, the limitations of 
the system should be carefully investigated, otherwise it might easily lead us to speculate about developing on-chip liver cancer spheroids using PDMS-glass microfluidic chip.

\section{REFERENCES}

[1] Liu H., Dang H., Wang XW., "The significance of intertumor and intratumor heterogeneity in liver cancer," Exp Mol Med. 2018, 5;50(1): e416.

[2] Friemel J., Rechsteiner M., Frick L., Böhm F., Struckmann K., Egger M., Moch H., Heikenwalder M., Weber A., "Intratumor heterogeneity in hepatocellular carcinoma," Clin Cancer Res. 2015, 15;21(8): 1951-61.

[3] Lu L. C., Hsu C. H., Hsu C., Chen A. L., "Tumor heterogeneity in hepatocellular carcinoma: facing the challenges," Liver Cancer. 2016, 5(2): 128-138.

[4] Mazzanti, R., Arena U, Tassi R., "Hepatocellular carcinoma: Where are we,” World J Exp Med. 2016, 6(1), 2136.

[5] Llovet, J. M., Ricci, S., Mazzaferro, V., Hilgard, P., Gane, E., Blanc, J., de Oliveira A. C., Santoro A., Raoul J. L., Forner A., Schwartz M., Porta C., Zeuzem S., Bolondi L., Greten T.F., Galle P.R., Seitz J.F., Borbath I., Häussinger D., Giannaris T., Shan M., Moscovici M., Voliotis D., Bruix J., "Sorafenib in Advanced Hepatocellular Carcinoma,"N Engl J Med. 2008, 359(4), 378-390.

[6] Roessler S., Budhu A., Wang X. W., "Deciphering cancer heterogeneity: the biological space," Front Cell Dev Biol. 2014, 2: 12.

[7] Elitas M., Brower K., Lu Y., Chen J. J., Fan R., “A microchip platform for interrogating tumor-macrophage paracrine signaling at the single-cell level," Lab Chip. 2014, 14(18), 3582-3588.

[8] Kang L., Chung B.G., Langer R., Khandemhosseini A., "Microfluidics for drug discovery and development: from target selection to product lifecycle management.” Drug Discov Today. 2008, 13 (1-2):1-13.

[9] Ma C., Fan R., Ahmad H., Shi Q., Comin-Anduix B., Chodon T., Koya R. C., Liu C. C., Kwong G. A., Radu C. G., Ribas A., Heath J. R. "A clinical microchip for evaluation of single immune cells reveal high functional heterogeneity in phenotypically similar T cells," Nature Medicine. 2011, 27: 738-744.

[10] Xiaohui C., Xiaoyue F., Qing X., Hua G., Ning Z. and Mingyue D., “Observation of liver cells in scanning probe acoustic microscope: A preliminary study,” Proc. SPIE 9790, 97901T (2016).

[11]Xia, Y., Whitesides, G. M., “Soft Lithography,” Annu. Rev. Mater. Sci. 1998, 37 (5): 551-575. 